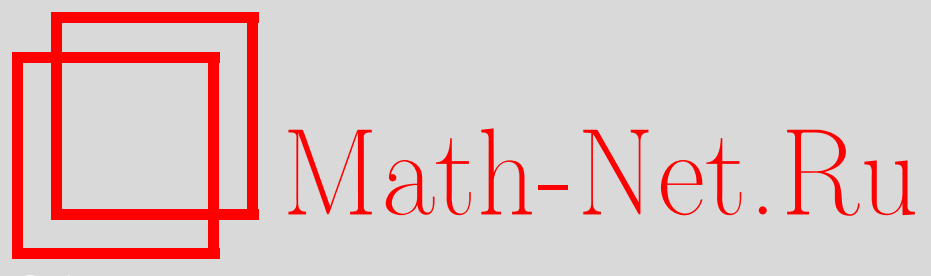

Ю. М. Дюкарев, Обобщенный критерий Стилтьеса полной неопределенности интерполяционных задач, $M a$ тем. заметки, 2008, том 84, выпуск 1, 23-39

DOI: https://doi.org/10.4213/mzm5192

Использование Общероссийского математического портала Math-Net.Ru подразумевает, что вы прочитали и согласны с пользовательским соглашением http://www . mathnet.ru/rus/agreement

Параметры загрузки:

IP : 52.6 .47 .48

26 апреля 2023 г., 11:34:22

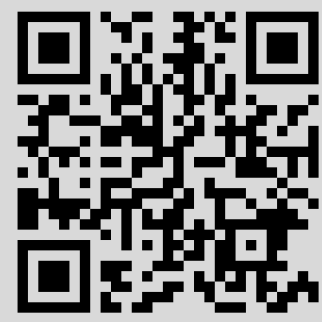


Том 84 выпуск 1 июль 2008

УДК 517.5

\section{Обобщенный критерий Стилтьеса полной неопределенности интерполяционных задач}

\section{Ю. М. Дюкарев}

Основным результатом этой статьи является обобщенный критерий Стилтьеса полной неопределенности интерполяционных задач в классе Стилтьеса. Этот критерий является обобщением для предельных интерполяционных проблем классического критерия неопределенности проблемы моментов, принадлежащего Стилтьесу. Он сформулирован в терминах параметров Стилтьеса $M_{j}$ и $N_{j}$. Мы получили явные формулы для параметров Стилтьеса. Общие построения проиллюстрированы на примерах проблемы моментов Стилтьеса и задачи Неванлинны-Пика в классе Стилтьеса.

Библиография: 11 названий.

1. Введение. Пусть дана бесконечная последовательность положительных вещественных чисел $s_{0}, s_{1}, \ldots, s_{n}, \ldots$ такая, что при всех $n \in \mathbb{N}$ матрицы Ганкеля $\left\{s_{j+k}\right\}_{j, k=0}^{n}$ и $\left\{s_{j+k+1}\right\}_{j, k=0}^{n-1}$ строго положительны. В проблеме моментов Стилтьеса требуется описать монотонно возрастающие функции $\sigma:[0, \infty) \rightarrow \mathbb{R}, \sigma(0)=0$, $\sigma(t+0)=\sigma(t), t \in[0, \infty)$, такие, что

$$
s_{j}=\int_{0}^{+\infty} t^{j} d \sigma(t) \quad \forall j \in \mathbb{N} \cup\{0\} .
$$

При сделанных предположениях у проблемы моментов (1) существует либо одно решение $\sigma(t)$, либо бесконечно много решений. Если существует бесконечно много решений, то проблема моментов (1) называется неопределенной. В классических работах Стилтьеса [1] был получен критерий неопределенности проблемы моментов. А именно, по заданным моментам $s_{j}$ с помощью определенной процедуры строятся две последовательности вещественных чисел $m_{k}>0, l_{k}>0, k \in \mathbb{N} \cup\{0\}$ (параметры Стилтьеса). Проблема моментов Стилтьеса неопределена тогда и только тогда, когда сходятся ряды

$$
\sum_{k=0}^{\infty} m_{k}, \quad \sum_{k=0}^{\infty} l_{k} .
$$

На случай матричной проблемы моментов Стилтьеса критерий (2) впервые был обобщен в статье [2].

С помощью теоремы Гамбургера-Неванлинны проблема моментов (1) может быть сведена к некоторой задаче интерполяционного типа для стилтьесовских функций

(C) Ю. М. ДюКАрев, 2008 
(см. [3; теорема 3.2.1]). Но в классе стилтьесовских функций существует много других интерполяционных задач, аналогичных той, которая участвует в теореме Гамбургера-Неванлинны. Это задачи Неванлинны-Пика и Каратеодори, многоточечная проблема моментов Нудельмана и другие интерполяционные задачи для стилтьесовских функций (см., например, [4]-[6]). Естественно предположить, что для этих интерполяционных задач должен существовать аналог критерия Стилтьеса (2). Однако до настоящего времени ни для одной интерполяционной задачи для стилтьесовских функций, отличной от проблемы моментов Стилтьеса, критерии такого типа получены не были.

Основной результат статьи доказан в теореме 3. В этой теореме критерий Стилтьеса (2) распространен на весьма общий случай предельной интерполяционной задачи (см. [7]) для стилтьесовских оператор-функций (ОФ). Предельная интерполяционная задача содержит в себе все основные интерполяционные задачи в классе Стилтьеса. Тем самым, установлена универсальная роль критериев стилтьесовского типа неопределенности интерполяционных задач в классе Стилтьеса. Общие построения проиллюстрированы на примерах задачи Неванлинны-Пика в классе Стилтьеса и проблемы моментов Стилтьеса. Из этих примеров видно, что критерий Стилтьеса в случае проблемы моментов является весьма простым частным случаем среди критериев стилтьесовского типа для других интерполяционных задач.

2. Предельная интерполяционная задача. В этом разделе приведены без доказательства необходимые для дальнейшего сведения о предельной интерполяционной задаче (подробное изложение и доказательства см. в [7]). Введем обозначения: $\mathbb{R}_{+}=\{x \in \mathbb{R}: x \geqslant 0\}, \mathbb{R}_{-}=\{x \in \mathbb{R}: x<0\}, \mathbb{C}_{+}=\{z \in \mathbb{C}: \operatorname{Im} z>0\}$, $\mathbb{C}_{-}=\{z \in \mathbb{C}: \operatorname{Im} z<0\}, \mathbb{C}_{ \pm}=\mathbb{C}_{+} \cup \mathbb{C}_{-}, \Pi_{-}=\{z \in \mathbb{C}: \operatorname{Re} z<0\}$

Пусть $\mathscr{G}_{1}, \mathscr{G}_{2}$ - сепарабельные и $\mathscr{H}$ - конечномерное гильбертовы пространства. Символом $\left\{\mathscr{G}_{1}, \mathscr{G}_{2}\right\}$ обозначим множество всех ограниченных линейных операторов, действующих из $\mathscr{G}_{1}$ в $\mathscr{G}_{2}$, символом $\left\{\mathscr{G}_{1}\right\}$ обозначим множество $\left\{\mathscr{G}_{1}, \mathscr{G}_{1}\right\}$, а символом $\left\{\mathscr{G}_{1}\right\}_{H}-$ множество ограниченных эрмитовых операторов в $\mathscr{G}_{1}$. Оператор $A \in\left\{\mathscr{G}_{1}\right\}_{H}$ называется неотрицательным, если $(f, A f) \geqslant 0 \forall f \in \mathscr{G}_{1}$. Множество неотрицательных операторов в $\mathscr{G}_{1}$ обозначим символом $\left\{\mathscr{G}_{1}\right\} \geqslant$. Неотрицательный оператор $A \in\left\{\mathscr{G}_{1}\right\} \geqslant$ называется строго положительным, если он обратим и $A^{-1} \in\left\{\mathscr{G}_{1}\right\}$. Множество строго положительных операторов в $\mathscr{G}_{1}$ обозначим символом $\left\{\mathscr{G}_{1}\right\}_{>}$. Пусть операторы $A, B \in\left\{\mathscr{G}_{1}\right\}_{H}$. Неравенство $A \geqslant B$ (соответственно $A>B$ ) означает, что $A-B \in\left\{\mathscr{G}_{1}\right\} \geqslant$ (соответственно $A-B \in\left\{\mathscr{G}_{1}\right\}_{>}$).

Тождественный и нулевой операторы, действующие в некотором гильбертовом пространстве $\mathscr{G}_{1}$, обозначим символами $I_{\mathscr{G}_{1}}$ и $0 \mathscr{G}_{1}$. Нулевой оператор, действующий из гильбертова пространства $\mathscr{G}_{1}$ в гильбертово пространство $\mathscr{G}_{2}$, обозначим символом $0 \mathscr{G}_{1} \mathscr{G}_{2}$. Когда из контекста ясно, о каких пространствах идет речь, то нижние индексы у единичного и тождественного операторов будем опускать.

Пусть заданы операторы $K_{1} \in\left\{\mathscr{G}_{1}\right\} \geqslant, K_{2} \in\left\{\mathscr{G}_{2}\right\} \geqslant, L_{1}, L_{2} \in\left\{\mathscr{G}_{2}, \mathscr{G}_{1}\right\}, v_{1} \in\left\{\mathscr{H}, \mathscr{G}_{1}\right\}$, $u_{2} \in\left\{\mathscr{H}, \mathscr{G}_{2}\right\}$. И пусть эти операторы удовлетворяют основному тождеству (ОТ)

$$
K_{1} L_{1}-L_{2} K_{2}=-v_{1} u_{2}^{*}
$$

Рассмотрим операторы

$$
T_{1}=L_{2} L_{1}^{*}, \quad T_{2}=L_{1}^{*} L_{2}, \quad u_{1}=L_{2} u_{2}, \quad v_{2}=L_{1}^{*} v_{1} .
$$


Пусть операторы $T_{r}$ таковы, что ОФ $R_{T_{r}}(z)=\left(I_{\mathscr{G}_{r}}-z T_{r}\right)^{-1}, r=1,2$, мероморфны в $\mathbb{C} \backslash \mathbb{R}_{+}$. Множество особых точек ОФ $R_{T_{1}}$ и $R_{T_{2}}$ в $\mathbb{C} \backslash \mathbb{R}_{+}$обозначим символом $\mathscr{Z}$. И пусть $\overline{\mathscr{Z}}=\{z \in \mathbb{C}: \bar{z} \in \mathscr{Z}\}$.

ОПредЕЛЕНиЕ 1. Голоморфная ОФ $s: \mathbb{C} \backslash \mathbb{R}_{+} \rightarrow\{\mathscr{H}\}$ называется стилтъесовской, если $\left\{s(z)-s^{*}(z)\right\} /\{z-\bar{z}\} \geqslant 0 \mathscr{H} \forall z \in \mathbb{C}_{ \pm}$и $s(x) \geqslant 0_{\mathscr{H}} \forall x \in \mathbb{R}_{-}$.

Класс всех стилтьесовских ОФ обозначим символом $\mathscr{S}$.

ОПРЕДЕЛЕниЕ 2. Упорядоченный набор операторов

$$
\mathscr{P}=\left\{K_{1}, L_{1}, v_{1}, K_{2}, L_{2}, u_{2}\right\},
$$

удовлетворяющих ОТ (3) и всем сформулированным выше условиям, называется обобщенной интерполяционной задачей стилтъесовского типа, а пространства $\mathscr{G}_{1}$, $\mathscr{G}_{2}, \mathscr{H}$ называются масштабными пространствами. При этом ОФ $s \in \mathscr{S}$ называется решением обобщенной интерполячионной задачи (5), если она удовлетворяет следующей системе основных матричных неравенств (OMH) Потапова $(r=1,2)$ :

$$
\left[\begin{array}{c|c}
K_{r} & R_{T_{r}}(z)\left\{v_{r} z^{r-1} s(z)-u_{r}\right\} \\
\hline * & \left\{z^{r-1} s(z)-\bar{z}^{r-1} s^{*}(z)\right\} /\{z-\bar{z}\}
\end{array}\right] \geqslant 0 \mathscr{G}_{r} \oplus \mathscr{H}, \quad z \in \mathbb{C}_{ \pm} \backslash \mathscr{Z} .
$$

Множество всех решений обобщенной интерполяционной задачи (5) обозначим символом $\mathscr{F}$.

Пусть заданы две обобщенные интерполяционные задачи

$$
\mathscr{P}^{(r)}=\left\{K_{1}^{(r)}, L_{1}^{(r)}, v_{1}^{(r)}, K_{2}^{(r)}, L_{2}^{(r)}, u_{2}^{(r)}\right\}, \quad r=1,2 .
$$

В обозначения объектов, относящихся к интерполяционной задаче $\mathscr{P}(1)$ (соответственно $\left.\mathscr{P}^{(2)}\right)$, будем добавлять верхний индекс (1) (соответственно (2)).

ОпредЕЛЕниЕ 3. Обобщенные интерполяционные задачи $\mathscr{P}^{(1)}$ и $\mathscr{P}^{(2)}$ называются унитарно эквивалентными, если существуют унитарные операторы

$$
\mathscr{U}_{1}: \mathscr{G}_{1}^{(1)} \rightarrow \mathscr{G}_{1}^{(2)}, \quad \mathscr{U}_{2}: \mathscr{G}_{2}^{(1)} \rightarrow \mathscr{G}_{2}^{(2)}
$$

такие, что

$$
\begin{array}{lll}
K_{1}^{(1)}=\mathscr{U}_{1}^{*} K_{1}^{(2)} \mathscr{U}_{1}, & L_{1}^{(1)}=\mathscr{U}_{1}^{*} L_{1}^{(2)} \mathscr{U}_{2}, & v_{1}^{(1)}=\mathscr{U}_{1}^{*} v_{1}^{(2)}, \\
K_{2}^{(1)}=\mathscr{U}_{2}^{*} K_{2}^{(2)} \mathscr{U}_{2}, & L_{2}^{(1)}=\mathscr{U}_{1}^{*} L_{2}^{(2)} \mathscr{U}_{2}, & u_{2}^{(1)}=\mathscr{U}_{2}^{*} u_{2}^{(2)} .
\end{array}
$$

Множества решений таких задач совпадают $\mathscr{F}^{(1)}=\mathscr{F}^{(2)}$.

Пусть дана обобщенная интерполяционная задача $(5)$, и пусть пространства $\mathscr{G}_{1}$ и $\mathscr{G}_{2}$ представлены в виде ортогональной суммы своих подпространств (подпространства $\widetilde{\mathscr{G}}_{r}$ предполагаются ненулевыми)

$$
\mathscr{G}_{r}=\widetilde{\mathscr{G}}_{r} \oplus \widehat{\mathscr{G}}_{r}, \quad r=1,2 .
$$

Пусть $\widetilde{P}_{r}$ обозначает оператор ортогонального проектирования на подпространство $\widetilde{\mathscr{G}}_{r}$ и выполнены условия

$$
L_{1} \widetilde{P}_{2}=\widetilde{P}_{1} L_{1} \widetilde{P}_{2}, \quad \widetilde{P}_{1} L_{2}=\widetilde{P}_{1} L_{2} \widetilde{P}_{2}
$$


В соответствии с $(10),(11)$ введем матричные обозначения $(r=1,2)$

$$
\begin{aligned}
& K_{r}=\left[\begin{array}{cc}
\widetilde{K}_{r} & B_{r} \\
B_{r}^{*} & C_{r}
\end{array}\right], \quad L_{1}=\left[\begin{array}{cc}
\widetilde{L}_{1} & D_{1} \\
0_{\widetilde{\mathscr{G}}_{2} \widehat{\mathscr{G}}_{1}} & \widehat{L}_{1}
\end{array}\right], \quad L_{2}=\left[\begin{array}{cc}
\widetilde{L}_{2} & 0_{\widehat{\mathscr{G}}_{2} \widetilde{\mathscr{G}}_{1}} \\
E_{2} & \widehat{L}_{2}
\end{array}\right], \\
& v_{1}=\left[\begin{array}{c}
\widetilde{v}_{1} \\
\check{v}_{1}
\end{array}\right], \quad u_{2}=\left[\begin{array}{c}
\widetilde{u}_{2} \\
\check{u}_{2}
\end{array}\right], \quad u_{1}=\left[\begin{array}{c}
\widetilde{L}_{2} \widetilde{u}_{2} \\
E_{2} \widetilde{u}_{2}+\widehat{L}_{2} \check{u}_{2}
\end{array}\right], \quad v_{2}=\left[\begin{array}{c}
\widetilde{L}_{1}^{*} \widetilde{v}_{1} \\
D_{1}^{*} \widetilde{v}_{1}+\widehat{L}_{1}^{*} \check{v}_{1}
\end{array}\right] \text {, } \\
& T_{1}=L_{2} L_{1}^{*}=\left[\begin{array}{cc}
\widetilde{L}_{2} \widetilde{L}_{1}^{*} & 0_{\widehat{\mathscr{G}}_{1} \widetilde{\mathscr{G}}_{1}} \\
E_{2} \widetilde{L}_{1}^{*}+\widehat{L}_{2} D_{1}^{*} & \widehat{L}_{2} \widehat{L}_{1}^{*}
\end{array}\right], \quad \widetilde{P}_{r}=\left[\begin{array}{cc}
I_{\widetilde{\mathscr{G}}_{r}} & 0_{\widehat{\mathscr{G}}_{r} \widetilde{\mathscr{G}}_{r}} \\
0_{\widetilde{\mathscr{G}}_{r} \widehat{\mathscr{G}}_{r}} & 0_{\widehat{\mathscr{G}}_{r}}
\end{array}\right], \\
& T_{2}=L_{1}^{*} L_{2}=\left[\begin{array}{cc}
\widetilde{L}_{1}^{*} \widetilde{L}_{2} & 0_{\widehat{\mathscr{G}}_{2} \widetilde{\mathscr{G}}_{2}} \\
D_{1}^{*} \widetilde{L}_{2}+\widehat{L}_{1}^{*} E_{2}^{*} & \widehat{L}_{1}^{*} L_{2}
\end{array}\right], \quad \widehat{P}_{r}=\left[\begin{array}{cc}
0_{\widetilde{\mathscr{G}}_{r}} & 0_{\widehat{\mathscr{G}}_{r} \widetilde{\mathscr{G}}_{r}} \\
0_{\widetilde{\mathscr{G}}_{r} \widehat{\mathscr{G}}_{r}} & I_{\widehat{\mathscr{G}}_{r}}
\end{array}\right] .
\end{aligned}
$$

Из ОТ (3) и представлений (12) следует индуцированное ОТ

$$
\widetilde{K}_{1} \widetilde{L}_{1}-\widetilde{L}_{2} \widetilde{K}_{2}=-\widetilde{v}_{1} \widetilde{u}_{2}^{*}
$$

Непосредственно убеждаемся в том, что операторы

$$
\widetilde{\mathscr{P}}=\left\{\widetilde{K}_{1}, \widetilde{L}_{1}, \widetilde{v}_{1}, \widetilde{K}_{2}, \widetilde{L}_{2}, \widetilde{u}_{2}\right\}
$$

и масштабные пространства $\left\{\widetilde{\mathscr{G}}_{1}, \widetilde{\mathscr{G}}_{2}, \mathscr{H}\right\}$ создают обобщенную интерполяционную задачу стилтьесовского типа.

ОПРЕДЕЛЕНИЕ 4. Интерполяционная задача (14) называется сужением интерполяционной задачи (5) на подпространства $\widetilde{\mathscr{G}}_{1}$ и $\widetilde{\mathscr{G}}_{2}$.

ОПРЕДЕЛЕНиЕ 5. Пусть даны две обобщенные интерполяционные задачи стилтьесовского типа

$$
\mathscr{P}^{(r)}=\left\{K_{1}^{(r)}, L_{1}^{(r)}, v_{1}^{(r)}, K_{2}^{(r)}, L_{2}^{(r)}, u_{2}^{(r)}\right\}, \quad r=1,2,
$$

с масштабными подпространствами $\mathscr{G}_{1}^{(r)}, \mathscr{G}_{2}^{(r)}$. Говорят, что интерполяционная задача $\mathscr{P}^{(1)}$ содержится в задаче $\mathscr{P}^{(2)}$, если $\mathscr{P}^{(1)}$ унитарно эквивалентна некоторому сужению $\widetilde{\mathscr{P}}^{(2)}$ интерполяционной задачи $\mathscr{P}^{(2)}$ на пару подпространств $\widetilde{\mathscr{G}}_{1}^{(2)} \subset \mathscr{G}_{1}^{(2)}$, $\widetilde{\mathscr{G}}_{2}^{(2)} \subset \mathscr{G}_{2}^{(2)}$

Коротко это записывается так $\mathscr{P}^{(1)} \subset \mathscr{P}^{(2)}$.

ОПРедЕлЕниЕ 6. Обобщенная интерполяционная задача (5) называется вполне неопределенной, если

$$
\begin{gathered}
K_{1} \in\left\{\mathscr{G}_{1}\right\}_{>}, \quad K_{2} \in\left\{\mathscr{G}_{2}\right\}_{>}, \\
v_{r} h=0 \in \mathscr{G}_{r} \forall h \in \mathscr{H} \quad \Leftrightarrow \quad h=0 \in \mathscr{H}, \quad r=1,2 . \\
u_{r} h=0 \in \mathscr{G}_{r} \forall h \in \mathscr{H} \quad \Leftrightarrow \quad h=0 \in \mathscr{H}, \quad
\end{gathered}
$$

Далее в этой статье мы будем рассматривать только вполне неопределенные обобщенные интерполяционные задачи.

Рассмотрим резольвентную матрицу задачи (5)

$$
U_{1}(z)=\left[\begin{array}{cc}
\alpha_{1} & \beta_{1} \\
\gamma_{1} & \delta_{1}
\end{array}\right]=\left[\begin{array}{c|c}
I_{\mathscr{H}}+z v_{2}^{*} R_{T_{2}^{*}}(z) K_{2}^{-1} u_{2} & -z v_{1}^{*} R_{T_{1}^{*}}(z) K_{1}^{-1} v_{1} \\
\hline u_{2}^{*} R_{T_{2}^{*}}(z) K_{2}^{-1} u_{2} & I_{\mathscr{H}}-z u_{1}^{*} R_{T_{1}^{*}}(z) K_{1}^{-1} v_{1}
\end{array}\right] .
$$


Введем операторы в пространстве $\mathscr{H} \oplus \mathscr{H}$

$$
J=\left[\begin{array}{cc}
0 \mathscr{H} & -i I_{\mathscr{H}} \\
i I_{\mathscr{H}} & 0_{\mathscr{H}}
\end{array}\right], \quad J_{\pi}=\left[\begin{array}{cc}
0_{\mathscr{H}} & I_{\mathscr{H}} \\
I_{\mathscr{H}} & 0_{\mathscr{H}}
\end{array}\right] .
$$

Из (3) и (4) следует, что $\forall z \in \mathbb{C} \backslash\left\{\mathbb{R}_{+} \cup \overline{\mathscr{Z}}\right\}$ выполнены равенства

$$
\begin{array}{r}
J-U_{1}(z) J U_{1}^{*}(z)=i(z-\bar{z})\left[\begin{array}{c}
v_{1}^{*} \\
u_{1}^{*}
\end{array}\right] R_{T_{1}^{*}}(z) K_{1}^{-1} R_{T_{1}^{*}}^{*}(z)\left[v_{1}, u_{1}\right], \\
U_{1}(z) J_{\pi} U_{1}^{*}(z)-J_{\pi}=-(z+\bar{z})\left[\begin{array}{c}
v_{1}^{*} \\
u_{1}^{*}
\end{array}\right] R_{T_{1}^{*}}(z) K_{1}^{-1} R_{T_{1}^{*}}^{*}(z)\left[v_{1}, u_{1}\right] \\
+2\left[\begin{array}{c}
z v_{2}^{*} \\
u_{2}^{*}
\end{array}\right] R_{T_{2}^{*}}(z) K_{2}^{-1} R_{T_{2}^{*}}^{*}(z)\left[\bar{z} v_{2}, u_{2}\right] .
\end{array}
$$

ОПРЕДЕЛЕНИЕ 7. Символом $\mathscr{W}_{s}$ обозначим множество всех мероморфных ОФ $U: \mathbb{C} \backslash \mathbb{R}_{+} \rightarrow \mathscr{H} \oplus \mathscr{H}$ таких, что

$$
\frac{U(z) J U^{*}(z)-J}{i(\bar{z}-z)} \geqslant 0 \quad \forall z \in \mathbb{C}_{ \pm} \backslash \mathscr{D}, \quad U(z) J_{\pi} U^{*}(z)-J_{\pi} \geqslant 0 \quad \forall z \in \Pi_{-} \backslash \mathscr{D} .
$$

Здесь $\mathscr{D}$ обозначает множество особых точек ОФ $U$ в $\mathbb{C} \backslash \mathbb{R}_{+}$.

Из (17) и (18) следует, что резольвентная матрица $U_{1} \in \mathscr{W}_{s}$.

Пусть $A, B \in\{\mathscr{H}\}_{>}$и выполнено условие $A \leqslant B$. Операторным интервалом $[A, B]$ называется

$$
[A, B]=\left\{\kappa \in\{\mathscr{H}\}_{>}: A \leqslant \kappa \leqslant B\right\} .
$$

Число $\operatorname{rank}\{B-A\}$ называется рангом операторного интервала. Интервал $[A, B]$ называется невырожденным, если $A<B$.

Через элементы резольвентной матрицы (16) записываются решения Фридрихса и Крейна обобщенной интерполяционной задачи (5)

$$
s_{F}(z)=\gamma_{1}(z) \alpha_{1}^{-1}(z) \in \mathscr{F}, \quad s_{K}(z)=\delta_{1}(z) \beta_{1}^{-1}(z) \in \mathscr{F} .
$$

Решения Фридрихса и Крейна можно записать в виде

$$
s_{F}(z)=u_{2}^{*}\left(K_{2}-z L_{1}^{*} K_{1} L_{1}\right)^{-1} u_{2}, \quad s_{K}(z)=\left\{v_{1}^{*}\left(L_{2} K_{2} L_{2}^{*}-z^{-1} K_{1}\right)^{-1} v_{1}\right\}^{-1} .
$$

Имеют место неравенства $s_{F}(x)<s_{K}(x), x \in \mathbb{R}_{-} \backslash \mathscr{Z}$.

Для всех $x \in \mathbb{R}_{-} \backslash \mathscr{Z}$ имеем

$$
U_{1}(x) J_{\pi} U_{1}^{*}(x)-J_{\pi}=2\left[\begin{array}{cc}
I_{\mathscr{H}} & 0_{\mathscr{H}} \\
s_{F}(x) & I_{\mathscr{H}}
\end{array}\right]\left[\begin{array}{cc}
\left(s_{K}(x)-s_{F}(x)\right)^{-1} & 0_{\mathscr{H}} \\
0 \mathscr{H} & s_{F}(x)
\end{array}\right]\left[\begin{array}{cc}
I_{\mathscr{H}} & s_{F}(x) \\
0 \mathscr{H} & I_{\mathscr{H}}
\end{array}\right] .
$$

ОПредЕЛЕниЕ 8. Невырожденный операторный интервал

$$
\mathscr{I}(x)=\left[s_{F}(x), s_{K}(x)\right], \quad x \in \mathbb{R}_{-} \backslash \mathscr{Z},
$$

называется интервалом Вейля в точке $x$. 
Пусть даны две обобщенные вполне неопределенные интерполяционные задачи $\mathscr{P}^{(1)}$ и $\mathscr{P}^{(2)}$, причем $\mathscr{P}^{(1)} \subset \mathscr{P}^{(2)}$. Пусть $\mathscr{Z}^{(k)}, \mathscr{F}^{(k)}, \mathscr{I}^{(k)}$ обозначают определенные выше объекты для интерполяционных задач $\mathscr{P}^{(k)}, k=1,2$. Тогда $\mathscr{F}^{(2)} \subset \mathscr{F}^{(1)}$ и $\mathscr{I}^{(2)}(x) \subset \mathscr{I}^{(1)}(x)$. Причем оба эти интервалы невырождены во всех точках $x \in$ $\mathbb{R}_{-} \backslash\left\{\mathscr{Z}^{(1)} \cup \mathscr{Z}^{(2)}\right\}$.

ОПРЕДЕЛЕНИЕ 9. Говорят, что задано упорядоченное семейство интерполяиионных задач стилтьесовского типа, если каждому $l \in \mathbb{N}$ поставлена в соответствие вполне неопределенная обобщенная интерполяционная задача стилтьесовского типа $\mathscr{P}^{(l)}$ так, что для любых $l_{1} \leqslant l_{2}$ выполнено условие $\mathscr{P}^{\left(l_{1}\right)} \subset \mathscr{P}^{\left(l_{2}\right)}$.

Обозначим символом $\left\{\mathscr{P}^{(l)}\right\}_{l \in \mathbb{N}}$ упорядоченное семейство интерполяционных задач. В этом контексте обобщенные интерполяционные задачи $\mathscr{P}^{(l)}$ называются $y с е-$ ченными интерполяционными задачами.

В обозначения объектов, связанных с семейством $\left\{\mathscr{P}^{(l)}\right\}_{l \in \mathbb{N}}$, введем верхний индекс $(l)$, который будет указывать, что соответствующие интервалы Вейля, резольвентные матрицы и т.д. связаны с $l$-й интерполяционной задачей.

ОПРЕДЕЛЕНИЕ 10. Пусть дано упорядоченное семейство интерполяционных задач $\left\{\mathscr{P}^{(l)}\right\}_{l \in \mathbb{N}}$, и пусть $\mathscr{F}^{(l)}$ обозначает множество всех решений интерполяционной задачи $\mathscr{P}^{(l)}$. ОФ $s \in \mathscr{S}$ называется решением предельной интерполяционной задачи, если $s \in \mathscr{F}^{(l)} \forall l \in \mathbb{N}$.

Множество решений предельной интерполяционной задачи обозначим символом $\mathscr{F}^{(\infty)}$, а саму предельную интерполяционную задачу - символом $\mathscr{P}(\infty)$.

Теорема 1. Пусть задано упорядоченное семейство обобщенных интерполяиионных задач стилтвесовского типа $\left\{\mathscr{P}^{(l)}\right\}_{l \in \mathbb{N}}$, и пусть $\mathscr{F}^{(l)}$ обозначает множество решений задачи $\mathscr{P}^{(l)}$, а $\mathscr{F}(\infty)$ - множество решений предельной интерполяционной задачи. И пусть, далее, $s_{F}^{(l)} u s_{K}^{(l)}$ являются решениями Фридрихса и Крейна задачи $\mathscr{P}^{(l)}$. Тогда

1) существуют равномерные на компактах $K \subset \mathbb{C} \backslash \mathbb{R}_{+}$предель

$$
s_{K}^{(\infty)}(z):=\lim _{l \rightarrow \infty} s_{K}^{(l)}(z) \in \mathscr{F}^{(\infty)}, \quad s_{F}^{(\infty)}(z):=\lim _{l \rightarrow \infty} s_{F}^{(l)}(z) \in \mathscr{F}^{(\infty)} ;
$$

2) для всех $s \in \mathscr{F}(\infty)$ выполняются неравенства

$$
0{ }_{\mathscr{H}}<s_{F}^{(\infty)}(x) \leqslant s(x) \leqslant s_{K}^{(\infty)}(x) \quad \forall x \in \mathbb{R}_{-} .
$$

ОПРЕДЕЛЕНИЕ 11. Операторный интервал

$$
\mathscr{I}^{(\infty)}(x):=\left[s_{F}^{(\infty)}(x), s_{K}^{(\infty)}(x)\right], \quad x \in \mathbb{R}_{-},
$$

называется предельным интервалом Вейля в точке $x \in \mathbb{R}_{-}$.

Пусть $l \in \mathbb{N}$. Напомним, что множество особых точек мероморфных ОФ $R_{T_{1}^{(l)}}$ и $R_{T_{2}^{(l)}}$ в $\mathbb{C} \backslash \mathbb{R}_{+}$мы обозначили символом $\mathscr{Z}^{(l)}$. Пусть $\mathscr{Z}(\infty):=\cup_{l \in \mathbb{N}} \mathscr{Z}^{(l)}$. С этого места и до конца статьи будем считать, что все предельные точки $\mathscr{Z}(\infty)$ принадлежат множеству $\mathbb{R}_{+} \cup\{\infty\}$. 
Лемма 1. Пусть дано $\left\{\mathscr{P}^{(l)}\right\}_{l \in \mathbb{N}}-$ упорядоченное семейство интерполяционных задач, и пусть $s_{K}^{(\infty)}, s_{F}^{(\infty)}$ обозначают решения Крейна и Фридрихса предельной интерполяционной задачи. Тогда для всех $x_{1}, x_{2} \in \mathbb{R}_{-} \backslash \mathscr{Z}^{(\infty)}$ имеет место равенство

$$
\operatorname{rank}\left\{s_{K}^{(\infty)}\left(x_{1}\right)-s_{F}^{(\infty)}\left(x_{1}\right)\right\}=\operatorname{rank}\left\{s_{K}^{(\infty)}\left(x_{2}\right)-s_{F}^{(\infty)}\left(x_{2}\right)\right\} .
$$

Другими словами, ранги предельных интервалов Вейля $\mathscr{I}^{(\infty)}(x)$ не зависят от выбора точки $x \in \mathbb{R}_{-} \backslash \mathscr{Z}^{(\infty)}$.

ОПредЕлЕниЕ 12 . Предельная интерполяционная задача $\mathscr{P}(\infty)$ называется вполне неопределенной, если все предельные интервалы Вейля

$$
\mathscr{I}^{(\infty)}(x)=\left[s_{F}^{(\infty)}(x), s_{K}^{(\infty)}(x)\right] \quad \forall x \in \mathbb{R}_{-} \backslash \mathscr{Z}^{(\infty)}
$$

являются невырожденными операторными интервалами.

\section{3. Обобщенный критерий Стилтьеса.}

ТЕорема 2. Пусть дана упорядоченная последовательность обобщенных вполне неопределенных интерполячионных задач стилтвесовского типа $\left\{\mathscr{P}^{(l)}\right\}_{l \in \mathbb{N}}, u$ пусть $\left\{U_{1}^{(l)}\right\}_{l \in \mathbb{N}}$ обозначает соответствующучо последовательность резольвентных матрии. Тогда существует принадлежсащая классу $\mathscr{W}_{s}$ последовательность $\mathrm{O} \Phi\left\{b_{k}\right\}_{k \in \mathbb{N}}$ такая, что

$$
U_{1}^{(l)}(z)=b_{1}(z) \times b_{2}(z) \times \cdots \times b_{l}(z) \quad \forall l \in \mathbb{N}, \quad \forall z \in \mathbb{C} \backslash\left\{\mathbb{R}_{+} \cup \overline{\mathscr{Z}}^{(l)}\right\} .
$$

ДокАЗАТЕЛЬСтво. Докажем теорему индукцией по $l$. При $l=1$ утверждение теоремы очевидно, так как $b_{1}(z)=U_{1}^{(1)}(z)$. Пусть формула (24) справедлива при $l>1$. По условию теоремы $\mathscr{P}^{(l)} \subset \mathscr{P}^{(l+1)}$. Поэтому интерполяционная задача $\mathscr{P}(l)$ унитарно эквивалентна сужению $\widetilde{P}^{(l+1)}$ интерполяционной задачи $\mathscr{P}(l+1)$ на пару подпространств $\widetilde{\mathscr{G}}_{1}^{(l+1)}$ и $\widetilde{\mathscr{G}}_{2}^{(l+1)}$. Пусть $U_{1}^{(l)}$ обозначает резольвентную матрицу обобщенной интерполяционной задачи $\mathscr{P}^{(l)}$, а $\widetilde{U}_{1}^{(l+1)}$ обозначает резольвентную матрицу обобщенной интерполяционной задачи $\widetilde{\mathscr{P}}^{(l+1)}$. Как было доказано в [7], резольвентные матрицы унитарно эквивалентных интерполяционных задач совпадают $U_{1}^{(l)}=\widetilde{U}_{1}^{(l+1)}$. В [8] доказано, что существует ОФ $b_{l+1}(z) \in \mathscr{W}_{s}$ такая, что $U_{1}^{(l+1)}(z)=\widetilde{U}_{1}^{(l+1)}(z) b_{l+1}(z)$. Отсюда

$$
U_{1}^{(l+1)}(z)=\widetilde{U}_{1}^{(l)}(z) b_{l+1}(z)=U_{1}^{(l)}(z) b_{l+1}(z)=b_{1}(z) b_{2}(z) \cdots b_{l}(z) b_{l+1}(z) .
$$

Отметим, что результаты из [8] нужно применить к ОФ $U_{1}^{(l+1)^{-1}}(z)=J U_{1}^{(l+1)^{*}}(\bar{z}) J$, а затем вернуться к ОФ $U_{1}^{(l+1)}(z)$.

Оператор $Q \in\{\mathscr{H} \oplus \mathscr{H}\}$ называется $J$-унитарным и $J_{\pi}$-растягивающим, если

$$
Q J Q^{*}-J=0 \mathscr{H} \oplus \mathscr{H} \quad \text { и } \quad Q J_{\pi} Q^{*}-J_{\pi} \geqslant 0 \mathscr{H} \oplus \mathscr{H}
$$

соответственно. В [9] доказано, что условия (25) эквивалентны условиям

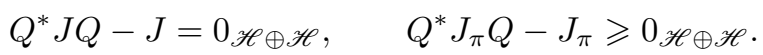

Легко видеть, что $J$-унитарный оператор обратим и что обратный оператор является $J$-унитарным. Кроме того, произведение $J$-унитарных операторов является $J$-унитарным оператором. 
Лемма 2. Пусть оператор $Q \in\{\mathscr{H} \oplus \mathscr{H}\}$ является $J$-унитарным и $J_{\pi}$-растягивающим. Тогда существует обратимый оператор $\widetilde{P} \in\{\mathscr{H}\}$ и неотрицательные операторы $\widetilde{M}, \widetilde{L} \in\{\mathscr{H}\} \geqslant$ maкuе, что

$$
Q=\left[\begin{array}{cc}
I & \widetilde{M} \\
0 & I
\end{array}\right]\left[\begin{array}{cc}
I & 0 \\
\widetilde{L} & I
\end{array}\right]\left[\begin{array}{cc}
\widetilde{P}^{*} & 0 \\
0 & \widetilde{P}^{-1}
\end{array}\right]
$$

Это представление единственно.

Доказательство. Рассмотрим матричное представление оператора $Q \in\{\mathscr{H} \oplus$ $\mathscr{H}\}$

$$
Q=\left[\begin{array}{ll}
Q_{11} & Q_{12} \\
Q_{21} & Q_{22}
\end{array}\right]
$$

В [10] доказано, что оператор $Q_{22}$ невырожден. Пусть

$$
\widetilde{P}=Q_{22}^{-1}, \quad \widetilde{L}=Q_{21} Q_{22}^{*}, \quad \widetilde{M}=Q_{12} Q_{22}^{-1}, \quad X=Q_{11} Q_{22}^{*}
$$

Тогда

$$
Q=\left[\begin{array}{cc}
X & \widetilde{M} \\
\widetilde{L} & I
\end{array}\right]\left[\begin{array}{cc}
\widetilde{P}^{*} & 0 \\
0 & \widetilde{P}^{-1}
\end{array}\right] .
$$

Оператор $Q$ в левой части (29) является $J$-унитарным и последний оператор в правой части (29) является $J$-унитарным. Отсюда следует, что и первый оператор в правой части (29) является $J$-унитарным. Из (25) и (26) имеем

$$
\left[\begin{array}{rr}
X & \widetilde{M} \\
\widetilde{L} & I
\end{array}\right] J\left[\begin{array}{rr}
X & \widetilde{M} \\
\widetilde{L} & I
\end{array}\right]^{*}=J, \quad\left[\begin{array}{cc}
X & \widetilde{M} \\
\widetilde{L} & I
\end{array}\right]^{*} J\left[\begin{array}{cc}
X & \widetilde{M} \\
\widetilde{L} & I
\end{array}\right]=J .
$$

Далее

$$
\begin{aligned}
& {\left[\begin{array}{cc}
-X \widetilde{M}^{*}+\widetilde{M} X^{*} & -X+\widetilde{M} \widetilde{L}^{*} \\
-\widetilde{L} \widetilde{M}^{*}+X^{*} & -\widetilde{L}+\widetilde{L}^{*}
\end{array}\right]=\left[\begin{array}{cc}
0 & -I \\
I & 0
\end{array}\right],} \\
& {\left[\begin{array}{cc}
-X^{*} \widetilde{L}+\widetilde{L}^{*} X & -X^{*}+\widetilde{L}^{*} \widetilde{M} \\
-\widetilde{M} * \widetilde{L}+X & -\widetilde{M}^{*}+\widetilde{M}
\end{array}\right]=\left[\begin{array}{cc}
0 & -I \\
I & 0
\end{array}\right] .}
\end{aligned}
$$

Из этих равенств следует, что

$$
\widetilde{M^{*}}=\widetilde{M}, \quad \widetilde{L}^{*}=\widetilde{L}, \quad X=I+\widetilde{M} \widetilde{L} .
$$

Теперь (29) можно записать в виде

$$
Q=\left[\begin{array}{cc}
I+\widetilde{M} \widetilde{L} & \widetilde{M} \\
\widetilde{L} & I
\end{array}\right]\left[\begin{array}{cc}
\widetilde{P}^{*} & 0 \\
0 & \widetilde{P}^{-1}
\end{array}\right]
$$

Отсюда следует (27). Единственность представления (27) очевидна. 
Покажем, что $\widetilde{M}, \widetilde{L} \in\{\mathscr{H}\} \geqslant$. Имеем

$$
\begin{aligned}
0_{\mathscr{H}} \leqslant Q J_{\pi} Q^{*}-J_{\pi} & =\left[\begin{array}{cc}
I+\widetilde{M} \widetilde{L} & \widetilde{M} \\
\widetilde{L} & I
\end{array}\right] J_{\pi}\left[\begin{array}{cc}
I+\widetilde{L} \widetilde{M} & L \\
\widetilde{M} & I
\end{array}\right]-J_{\pi} \\
& =2\left[\begin{array}{cc}
\widetilde{M}+\widetilde{M} \widetilde{L} \widetilde{M} & \widetilde{M} \widetilde{L} \\
\widetilde{L} \widetilde{M} & \widetilde{L}
\end{array}\right] .
\end{aligned}
$$

Отсюда следует, что $\widetilde{L} \in\{\mathscr{H}\} \geqslant$. Аналогичным образом, из неравенства $0 \mathscr{H} \leqslant$ $Q^{*} J_{\pi} Q-J_{\pi}$ следует, что $\widetilde{M} \in\{\mathscr{H}\} \geqslant$.

Пусть дана упорядоченная последовательность обобщенных вполне неопределенных интерполяционных задач стилтьесовского типа $\left\{\mathscr{P}^{(l)}\right\}_{l \in \mathbb{N}}$, и пусть все предельные точки множества $\mathscr{Z}(\infty)=\bigcup_{l \in \mathbb{N}} \mathscr{Z}^{(l)}$ принадлежат $\mathbb{R}_{+} \cup\{\infty\}$. Сомножители в правой части (24) принадлежат классу $\mathscr{W}_{s}$. Поэтому в произвольной точке $x_{0} \in \mathbb{R}_{-} \backslash \mathscr{Z}^{(\infty)}$ операторы $\left\{b_{k}\left(x_{0}\right)\right\}_{k \in \mathbb{N}}$ удовлетворяют условиям леммы 2 (см. определение 7). Следовательно, для всех $k \in \mathbb{N}$ существуют невырожденные операторы $\widetilde{P}^{(k)}\left(x_{0}\right) \in\{\mathscr{H}\}$ и операторы $\widetilde{M}^{(k)}\left(x_{0}\right), \widetilde{L}^{(k)}\left(x_{0}\right) \in\{\mathscr{H}\} \geqslant$ такие, что $(\forall l \in \mathbb{N})$

$$
\begin{aligned}
& U_{1}^{(l)}\left(x_{0}\right)=\left[\begin{array}{cc}
I & \widetilde{M}^{(1)}\left(x_{0}\right) \\
0 & I
\end{array}\right]\left[\begin{array}{cc}
I & 0 \\
\widetilde{L}^{(1)}\left(x_{0}\right) & I
\end{array}\right]\left[\begin{array}{cc}
\widetilde{P}^{(1)^{*}}\left(x_{0}\right) & 0 \\
0 & \widetilde{P}^{(1)^{-1}}\left(x_{0}\right)
\end{array}\right] \\
& \times\left[\begin{array}{cc}
I & \widetilde{M}^{(2)}\left(x_{0}\right) \\
0 & I
\end{array}\right]\left[\begin{array}{cc}
I & 0 \\
\widetilde{L}^{(2)}\left(x_{0}\right) & I
\end{array}\right]\left[\begin{array}{cc}
\widetilde{P}^{(2)^{*}}\left(x_{0}\right) & 0 \\
0 & \widetilde{P}^{(2)^{-1}}\left(x_{0}\right)
\end{array}\right] \times \cdots \\
& \times\left[\begin{array}{cc}
I & \widetilde{M}^{(l)}\left(x_{0}\right) \\
0 & I
\end{array}\right]\left[\begin{array}{cc}
I & 0 \\
\widetilde{L}^{(l)}\left(x_{0}\right) & I
\end{array}\right]\left[\begin{array}{cc}
\widetilde{P}^{(l)^{*}}\left(x_{0}\right) & 0 \\
0 & \widetilde{P}^{(l)^{-1}}\left(x_{0}\right)
\end{array}\right] .
\end{aligned}
$$

Введем три бесконечные последовательности операторов

$$
\begin{aligned}
& M^{(1)}\left(x_{0}\right)=\widetilde{M}^{(1)}\left(x_{0}\right), \\
& M^{(2)}\left(x_{0}\right)=\widetilde{P}^{(1)^{*}}\left(x_{0}\right) \widetilde{M}^{(2)}\left(x_{0}\right) \widetilde{P}^{(1)}\left(x_{0}\right), \\
& M^{(k)}\left(x_{0}\right)=\widetilde{P}^{(1)^{*}}\left(x_{0}\right) \cdots \widetilde{P}^{(k-1)^{*}}\left(x_{0}\right) \widetilde{M}^{(k)}\left(x_{0}\right) \widetilde{P}^{(k-1)}\left(x_{0}\right) \cdots \widetilde{P}^{(1)}\left(x_{0}\right), \\
& L^{(1)}\left(x_{0}\right)=\widetilde{L}^{(1)}\left(x_{0}\right), \\
& L^{(2)}\left(x_{0}\right)=\left\{\widetilde{P}^{(1)^{-1}}\left(x_{0}\right)\right\} \widetilde{L}^{(2)}\left(x_{0}\right)\left\{\widetilde{P}^{(1)^{-1}}\left(x_{0}\right)\right\}^{*}, \\
& L^{(k)}\left(x_{0}\right)=\left\{\widetilde{P}^{(1)^{-1}}\left(x_{0}\right) \cdots \widetilde{P}^{(k-1)^{-1}}\left(x_{0}\right)\right\} \widetilde{L}^{(k)}\left(x_{0}\right)\left\{\widetilde{P}^{(1)^{-1}}\left(x_{0}\right) \cdots \widetilde{P}^{(k-1)^{-1}}\left(x_{0}\right)\right\}^{*}, \\
& P^{(1)^{*}}\left(x_{0}\right)=\widetilde{P}^{(1)^{*}}\left(x_{0}\right), \\
& P^{(2)^{*}}\left(x_{0}\right)=\widetilde{P}^{(1)^{*}}\left(x_{0}\right) \widetilde{P}^{(2)^{*}}\left(x_{0}\right), \\
& P^{(k)^{*}}\left(x_{0}\right)=\widetilde{P}^{(1)^{*}}\left(x_{0}\right) \cdots \widetilde{P}^{(k)^{*}}\left(x_{0}\right),
\end{aligned}
$$


ОПРЕДЕЛЕНИЕ 13. Операторы $M^{(k)}\left(x_{0}\right)$ и $L^{(k)}\left(x_{0}\right)$ называются обобщенными параметрами Стилтъеса, ассоциированными с упорядоченной последовательностью обобщенных вполне неопределенных интерполяционных задач стилтьесовского типа $\left\{\mathscr{P}^{(l)}\right\}_{l \in \mathbb{N}}$.

Последовательно переставляя в (30) блочно диагональные операторы слева направо и учитывая $(31)$, получим $(\forall l \in \mathbb{N})$

$$
\begin{aligned}
U_{1}^{(l)}\left(x_{0}\right)=\left[\begin{array}{cc}
I & M^{(1)}\left(x_{0}\right) \\
0 & I
\end{array}\right]\left[\begin{array}{cc}
I & 0 \\
L^{(1)}\left(x_{0}\right) & I
\end{array}\right]\left[\begin{array}{cc}
I & M^{(2)}\left(x_{0}\right) \\
0 & I
\end{array}\right]\left[\begin{array}{cc}
I & 0 \\
L^{(2)}\left(x_{0}\right) & I
\end{array}\right] \times \cdots \\
\times\left[\begin{array}{cc}
I & M^{(l)}\left(x_{0}\right) \\
0 & I
\end{array}\right]\left[\begin{array}{cc}
I & 0 \\
L^{(l)}\left(x_{0}\right) & I
\end{array}\right]\left[\begin{array}{cc}
P^{(l)^{*}}\left(x_{0}\right) & 0 \\
0 & P^{(l)^{-1}}\left(x_{0}\right)
\end{array}\right] .
\end{aligned}
$$

Причем

$$
M^{(k)}\left(x_{0}\right) \in\{\mathscr{H}\}_{\geqslant}, \quad L^{(k)}\left(x_{0}\right) \in\{\mathscr{H}\} \geqslant \quad \forall k \in \mathbb{N} .
$$

Вместе с произведением (32) будем рассматривать еще и произведение

$$
\begin{gathered}
\mathfrak{U}_{1}^{(l)}\left(x_{0}\right)=\left[\begin{array}{cc}
I & M^{(1)}\left(x_{0}\right) \\
0 & I
\end{array}\right]\left[\begin{array}{cc}
I & 0 \\
L^{(1)}\left(x_{0}\right) & I
\end{array}\right]\left[\begin{array}{cc}
I & M^{(2)}\left(x_{0}\right) \\
0 & I
\end{array}\right]\left[\begin{array}{cc}
I & 0 \\
L^{(2)}\left(x_{0}\right) & I
\end{array}\right] \times \cdots \\
\times\left[\begin{array}{cc}
I & M^{(l)}\left(x_{0}\right) \\
0 & I
\end{array}\right]\left[\begin{array}{cc}
I & 0 \\
L^{(l)}\left(x_{0}\right) & I
\end{array}\right] .
\end{gathered}
$$

Из (32) и (34) следует, что

$$
U_{1}^{(l)}\left(x_{0}\right)=\mathfrak{U}_{1}^{(l)}\left(x_{0}\right)\left[\begin{array}{cc}
P^{(l)^{*}}\left(x_{0}\right) & 0 \\
0 & P^{(l)^{-1}}\left(x_{0}\right)
\end{array}\right] .
$$

Введем матрицы

$$
H_{2 k-1}\left(x_{0}\right)=\left[\begin{array}{cc}
0 & M^{(k)}\left(x_{0}\right) \\
0 & 0
\end{array}\right], \quad H_{2 k}\left(x_{0}\right)=\left[\begin{array}{cc}
0 & 0 \\
L^{(k)}\left(x_{0}\right) & 0
\end{array}\right], \quad k \in \mathbb{N} .
$$

Легко видеть, что $\mathfrak{U}_{1}^{(l)}\left(x_{0}\right)$ можно записать в виде

$$
\mathfrak{U}_{1}^{(l)}\left(x_{0}\right)=\prod_{k=1}^{2 l} e^{H_{k}\left(x_{0}\right)} .
$$

В операторных произведениях считаем, что индексы сомножителей возрастают в порядке слева направо.

ТЕОРЕма 3. Пусть дана упорядоченная последовательность обобщенных вполне неопределенных интерполяиионных задач стилтьесовского типа $\left\{\mathscr{P}^{(l)}\right\}_{l \in \mathbb{N}}, u$ пусть через $\mathscr{P}(\infty)$ обозначена соответствующая предельная интерполячионная задача. Пусть, далее, произвольная точка $x_{0} \in \mathbb{R}_{-} \backslash \mathscr{Z}(\infty)$ и операторы $M^{(k)}\left(x_{0}\right)$ и $L^{(k)}\left(x_{0}\right)$ являются ассочиированными параметрами Стилтвеса. Для того, итобъ предельная интерполяционная задача $\mathscr{P}^{(\infty)}$ была вполне неопределенной, необходимо и достаточно, чтобы сходились два ряда

$$
\sum_{k=1}^{\infty} M^{(k)}\left(x_{0}\right) \quad u \quad \sum_{k=1}^{\infty} L^{(k)}\left(x_{0}\right) .
$$


ДоКАЗАТЕЛЬСТвО. Пусть предельная интерполяционная задача является вполне неопределенной и произвольная точка $x_{0} \in \mathbb{R}_{-} \backslash \mathscr{Z}(\infty)$. Покажем, что $\forall l, k \in \mathbb{N}$ выполнены следующие три соотношения:

$$
\left\|\mathfrak{U}_{1}^{(l)}\left(x_{0}\right) J_{\pi} \mathfrak{U}_{1}^{(l) *}\left(x_{0}\right)-J_{\pi}\right\| \leqslant C, \quad \operatorname{det}\left[\mathfrak{U}_{1}^{(l)}\left(x_{0}\right)\right]=1, \quad H_{k}\left(x_{0}\right) J_{\pi} \geqslant 0 .
$$

Здесь $C \in \mathbb{R}_{+}-$некоторая константа. Действительно, из (35) следует, что $J_{\pi}$-формы ОФ $U_{1}^{(l)}$ и $\mathfrak{U}_{1}^{(l)}$ совпадают. Отсюда и из $(20)$ имеем

$$
\begin{aligned}
\mathfrak{U}_{1}^{(l)}\left(x_{0}\right) J_{\pi} \mathfrak{U}_{1}^{(l)^{*}}\left(x_{0}\right)-J_{\pi} \\
=U_{1}^{(l)}\left(x_{0}\right) J_{\pi} U_{1}^{(l)^{*}}\left(x_{0}\right)-J_{\pi} \\
\quad=\left[\begin{array}{cc}
I_{\mathscr{H}} & 0 \\
s_{F}^{(l)}\left(x_{0}\right) & I_{\mathscr{H}}
\end{array}\right]\left[\begin{array}{cc}
\left(s_{K}^{(l)}\left(x_{0}\right)-s_{F}^{(l)}\left(x_{0}\right)\right)^{-1} & 0 \\
0_{\mathscr{H}} & s_{F}^{(l)}\left(x_{0}\right)
\end{array}\right]\left[\begin{array}{cc}
I_{\mathscr{H}} & s_{F}^{(l)}\left(x_{0}\right) \\
0_{\mathscr{H}} & I_{\mathscr{H}}
\end{array}\right] .
\end{aligned}
$$

Из этой формулы и из $(21),(23)$ и определения 12 следует сходимость $J_{\pi}$-формы $\mathfrak{U}_{1}^{(l)}\left(x_{0}\right) J_{\pi} \mathfrak{U}_{1}^{(l)^{*}}\left(x_{0}\right)-J_{\pi}$ при $l \rightarrow \infty$. Отсюда следует первое из соотношений $(39)$. Равенство в (39) следует из (34). Из (36) и (33) имеем

$$
H_{2 k-1}\left(x_{0}\right) J_{\pi}=\left[\begin{array}{cc}
M^{(k)}\left(x_{0}\right) & 0 \\
0 & 0
\end{array}\right] \geqslant 0, \quad H_{2 k}\left(x_{0}\right) J_{\pi}=\left[\begin{array}{cc}
0 & 0 \\
0 & L^{(k)}\left(x_{0}\right)
\end{array}\right] \geqslant 0 .
$$

Доказаны все соотношения (39). По теореме Потапова [11] отсюда следует сходимость операторного произведения $\prod_{k=0}^{\infty} e^{H_{k}\left(x_{0}\right)}$ и ряда $\sum_{k=0}^{\infty} H_{k}\left(x_{0}\right)$. Из сходимости последнего ряда и из вида $H_{k}\left(x_{0}\right)$ следует сходимость рядов $(38)$.

Наоборот, пусть сходятся ряды (38). Отсюда и из (36) следует сходимость ряда $\sum_{k=1}^{\infty} H_{k}\left(x_{0}\right)$. Поэтому (см. [11]) существует предел бесконечного операторного произведения

$$
\mathfrak{U}_{1}^{(\infty)}\left(x_{0}\right)=\lim _{l \rightarrow \infty} \mathfrak{U}_{1}^{(l)}\left(x_{0}\right)=\lim _{l \rightarrow \infty} e^{H_{1}\left(x_{0}\right)} e^{H_{2}\left(x_{0}\right)} \cdots e^{H_{2 l}\left(x_{0}\right)} .
$$

Из (40) имеем

$$
\begin{aligned}
& {\left[\begin{array}{cc}
s_{K}^{(l)}\left(x_{0}\right)-s_{F}^{(l)}\left(x_{0}\right) & 0 \\
0 & s_{F}^{(l)^{-1}}\left(x_{0}\right)
\end{array}\right]\left[\begin{array}{cc}
I & 0 \\
-s_{F}^{(l)}\left(x_{0}\right) & I
\end{array}\right]\left[\mathfrak{U}_{1}^{(l)}\left(x_{0}\right) J_{\pi} \mathfrak{U}_{1}^{(l)^{*}}\left(x_{0}\right)-J_{\pi}\right]} \\
& \quad=2\left[\begin{array}{cc}
I & s_{F}^{(l)}\left(x_{0}\right) \\
0 & I
\end{array}\right] .
\end{aligned}
$$

В этом равенстве все операторы имеют пределы при $l \rightarrow \infty$, а предел правой части является обратимым оператором. Поэтому $s_{K}^{(\infty)}\left(x_{0}\right)-s_{F}^{(\infty)}\left(x_{0}\right)>0$. Отсюда, из леммы 1 и определения 12 следует, что предельная интерполяционная задача является вполне неопределенной.

Теорема 3 является обобщением критерия Стилтьеса на широкий класс интерполяционных задач для стилтьесовских ОФ.

ЗАмЕчАниЕ. Из доказанной теоремы и результатов статей [2] и [7] следует, что при условии полной неопределенности предельной интерполяционной задачи существуют пределы

$$
\mathfrak{U}_{1}^{(\infty)}(z)=\lim _{l \rightarrow \infty} \mathfrak{U}_{1}^{(l)}(z) \quad \forall z \in \mathbb{C} \backslash\left\{\mathbb{R}_{+} \cup \mathscr{Z}^{(\infty)}\right\} .
$$


Эти пределы являются равномерными на компактах $K \subset \mathbb{C} \backslash\left\{\mathbb{R}_{+} \cup \mathscr{Z}(\infty)\right\}$ и ОФ $\mathfrak{U}_{1}^{(\infty)}$ является резольвентной матрицей предельной интерполяционной задачи.

\section{4. Примеры.}

4.1. Задача Неванлинны-Пика в классе Стилтьеса. В задаче Неванлинны-Пика задана бесконечная последовательность попарно различных комплексных чисел $z_{1}$, $z_{2}, \ldots, z_{k}, \ldots, z_{j} \neq \bar{z}_{k}, j, k \in \mathbb{N}$, и бесконечная последовательность операторов $s_{1}, s_{2}$, $\ldots, s_{k}, \ldots$, действующих в пространстве в $\mathscr{H}$. Требуется описать множество стилтьесовских ОФ $s: \mathbb{C} \backslash \mathbb{R}_{+} \rightarrow\{\mathscr{H}\}$ таких, что

$$
s\left(z_{j}\right)=s_{j} \quad \forall j \in \mathbb{N} .
$$

Нас будет интересовать неопределенный случай, когда задача (41) имеет бесконечно много решений . Поэтому мы считаем, что выполнено необходимое условие неопределенности - предельные точки последовательности $\left\{z_{j}\right\}_{j \in \mathbb{N}}$ принадлежат множеству $\mathbb{R}_{+} \cup\{\infty\}$.

Вместе с задачей (41) с бесконечным числом узлов интерполяции будем рассматривать и усеченные задачи Неванлинны-Пика. В таких задачах фиксируется число $l \in \mathbb{N}$ и требуется описать множество ОФ $s: \mathbb{C} \backslash \mathbb{R}_{+} \rightarrow\{\mathscr{H}\}$ таких, что

$$
s\left(z_{j}\right)=s_{j}, \quad 1 \leqslant j \leqslant l, \quad s \in \mathscr{S} .
$$

В [7] показано, что усеченную задачу (42) можно рассматривать как обобщенную интерполяционную задачу стилтьесовского типа. Масштабные пространства имеют вид

$$
\mathscr{G}_{1}^{(l)}=\mathscr{G}_{2}^{(l)}=\underbrace{\mathscr{H} \oplus \mathscr{H} \oplus \cdots \oplus \mathscr{H}}_{l \text { слагаемых }}, \quad \mathscr{H} .
$$

Операторы $K_{1}^{(l)}, L_{1}^{(l)}, v_{1}^{(l)}, K_{2}^{(l)}, L_{2}^{(l)}, u_{2}^{(l)}$, участвующие в обобщенной интерполяционной задаче (5), зададим естественными матричными представлениями

$$
\begin{aligned}
& L_{1}^{(l)}=\operatorname{diag}\left\{I_{\mathscr{H}}, \ldots, I_{\mathscr{H}}\right\} \in\left\{\mathscr{G}_{1}^{(l)}\right\}, \quad v_{1}^{(l)}=\operatorname{col}\left\{I_{\mathscr{H}}, \ldots, I_{\mathscr{H}}\right\} \in\left\{\mathscr{H}, \mathscr{G}_{1}^{(l)}\right\}, \\
& L_{2}^{(l)}=\operatorname{diag}\left\{z_{1}^{-1} I_{\mathscr{H}}, \ldots, z_{l}^{-1} I_{\mathscr{H}}\right\} \in\left\{\mathscr{G}_{2}^{(l)}\right\}, \quad u_{2}^{(l)}=L_{2}^{(l)^{-1}} \cdot \operatorname{col}\left\{s_{1}, \ldots, s_{l}\right\} \in\left\{\mathscr{H}, \mathscr{G}_{2}\right\} . \\
& K_{r}^{(l)}=L_{2}^{(l)}{ }^{-1} \cdot\left\{\frac{z_{i}^{r-1} s_{i}-\bar{z}_{j}^{r-1} s_{j}^{*}}{z_{i}-\bar{z}_{j}}\right\}_{i, j=1, \ldots, l} \cdot L_{2}^{(l)^{-1^{*}}} \in\left\{G_{r}\right\}, \quad r=1,2 .
\end{aligned}
$$

Отсюда и из (4) имеем

$$
T_{1}^{(l)}=T_{2}^{(l)}=L_{2}^{(l)}, \quad v_{2}^{(l)}=v_{1}^{(l)}, \quad u_{1}^{(l)}=\operatorname{col}\left\{s_{1}, \ldots, s_{l}\right\} .
$$

Введем обозначение

$$
T^{(l)}=T_{1}^{(l)}=T_{2}^{(l)}, \quad v^{(l)}=v_{2}^{(l)}=v_{1}^{(l)} .
$$

Очевидно, что выполнено ОТ (3). Необходимым и достаточным условием разрешимости задачи (42) являются неравенства $K_{1}^{(l)} \geqslant 0, K_{2}^{(l)} \geqslant 0$. Более того (см. [7]), ОФ $s \in \mathscr{S}$ является решением усеченной задачи (42) тогда и только тогда, когда 
она удовлетворяет системе ОМН (6). Таким образом, множество решений интерполяционной задачи (41) совпадает с множеством решений интерполяционной задачи стилтьесовского типа $\mathscr{P}(l)=\left\{K_{1}^{(l)}, L_{1}^{(l)}, v_{1}^{(l)}, K_{2}^{(l)}, L_{2}^{(l)}, u_{2}^{(l)}\right\}$. Условиями полной неопределенности являются условия $K_{1}^{(l)}>0, K_{2}^{(l)}>0$. Легко видеть, что при этом все остальные условия в (15) выполнены, т.е. задача (42) является вполне неопределенной.

Пусть при всех $l \in \mathbb{N}$ интерполяционные задачи (42) являются вполне неопределенными. В [7] показано, что интерполяционные задачи (42) являются упорядоченным семейством. При этом предельная интерполяционная задача совпадает с (41).

Получим явные формулы для параметров Стилтьеса. Для этого при всех $l \in \mathbb{N}$ рассмотрим резольвентные матрицы задач (42)

$$
U_{1}^{(l)}(z)=\left[\begin{array}{c|c}
I_{\mathscr{H}}+z v^{(l)^{*}} R_{T^{(l)}}(z) K_{2}^{(l)^{-1}} u_{2}^{(l)} & -z v^{(l)^{*}} R_{T^{(l)}}(z) K_{1}^{(l)^{-1}} v^{(l)} \\
\hline u_{2}^{(l)^{*}} R_{T^{(l)}}(z) K_{2}^{(l)^{-1}} u_{2}^{(l)} & I_{\mathscr{H}}-z u_{1}^{(l)^{*}} R_{T^{(l)}}(z) K_{1}^{(l)^{-1}} v^{(l)}
\end{array}\right] .
$$

При $l \geqslant 2$ с каждой усеченной задачей Неванлинны-Пика (42) свяжем следующие объекты $(r=1,2)$ :

$$
\begin{aligned}
& B_{r}^{(l)}=T^{(l-1)^{-1}} \cdot\left[\begin{array}{c}
\frac{z_{1}^{r-1} s_{1}-\bar{z}_{l}^{r-1} s_{l}^{*}}{z_{1}-\bar{z}_{l}} \\
\vdots \\
\frac{z_{l-1}^{r-1} s_{l-1}-\bar{z}_{l}^{r-1} s_{l}^{*}}{z_{l-1}-\bar{z}_{l}}
\end{array}\right] \cdot \bar{z}_{l} I_{\mathscr{H}}, \\
& \widehat{v}^{(l)}=B_{1}^{(l)^{*}} K_{1}^{(l-1)^{-1}} v^{(l-1)}-I_{\mathscr{H}}, \\
& \widehat{u}_{2}^{(l)}=-B_{2}^{(l)^{*}} K_{2}^{(l-1)^{-1}} u_{2}^{(l-1)}+z_{l} s_{l}, \quad \widehat{u}_{1}^{(l)}=-z_{l}^{-1} B_{2}^{(l)^{*}} K_{2}^{(l-1)^{-1}} u_{2}^{(l-1)}+s_{l}, \\
& \widehat{K}_{r}^{(l)}=\frac{z_{l}^{r-1} s_{l}-\bar{z}_{l}^{r-1} s_{l}^{*}}{z_{l}-\bar{z}_{l}}-B_{r}^{(l)^{*}} K_{r}^{(l-1)^{-1}} B_{r}^{(l)}>0_{\widehat{\mathscr{G}}_{r}} .
\end{aligned}
$$

Введем элементарные множители Бляшке-Потапова $(k \geqslant 2)$

$$
\begin{gathered}
b_{1}(z)=I_{\mathscr{H} \oplus \mathscr{H}}+\frac{1}{1-z \bar{z}_{1}^{-1}}\left[\begin{array}{c|c|c}
+z v^{(1)^{*}} K_{2}^{(1)^{-1}} u_{2}^{(1)} & -z v^{(1)^{*}} K_{1}^{(1)^{-1}} v^{(1)} \\
\hline u_{2}^{(1)^{*}} K_{2}^{(1)^{-1}} u_{2}^{(1)} & -z u_{1}^{(1)^{*}} K_{1}^{(1)^{-1}} v^{(1)}
\end{array}\right], \\
b_{k}(z)=I_{\mathscr{H} \oplus \mathscr{H}}+\frac{1}{1-z \bar{z}_{k}^{-1}}\left[\begin{array}{c|c|c}
+z \widehat{v}^{(k)^{*}} \widehat{K}_{2}^{(k)^{-1}} \widehat{u}_{2}^{(k)} & -z \widehat{v}^{(k)^{*}} \widehat{K}_{1}^{(k)^{-1}} \widehat{v}^{(k)} \\
\hline \widehat{u}_{2}^{(k)^{*}} \widehat{K}_{2}^{(k)^{-1}} \widehat{u}_{2}^{(k)} & -z \widehat{u}_{1}^{(k)^{*}} \widehat{K}_{1}^{(k)^{-1}} \widehat{v}^{(k)}
\end{array}\right] .
\end{gathered}
$$

В [8] доказано, что резольвентные матрицы (43) представимы в виде произведения множителей Бляшке-Потапова (44)

$$
U_{1}^{(l)}(z)=b_{1}(z) \cdot b_{2}(z) \cdots b_{l}(z) \quad \forall l \in \mathbb{N} .
$$

Элементарные множители Бляшке-Потапова (44) принадлежат классу $\mathscr{W}_{s}$. Зафиксируем произвольную точку $x_{0} \in \mathbb{R}$. В этой точке все множители Бляшке-Потапова $b_{k}\left(x_{0}\right)$ удовлетворяют условиям леммы 2 и допускают представления $(27)$

$$
b_{k}\left(x_{0}\right)=\left[\begin{array}{cc}
I & \widetilde{M}^{(k)}\left(x_{0}\right) \\
0 & I
\end{array}\right]\left[\begin{array}{cc}
I & 0 \\
\widetilde{L}^{(k)}\left(x_{0}\right) & I
\end{array}\right]\left[\begin{array}{cc}
\widetilde{P}^{(k)^{*}}\left(x_{0}\right) & 0 \\
0 & \widetilde{P}^{(k)^{-1}}\left(x_{0}\right)
\end{array}\right] .
$$


Простые вычисления по формулам (28) приводят к следующим выражениям для операторов из $(46)(k \geqslant 2)$ :

$$
\begin{aligned}
\widetilde{P}^{(1)}\left(x_{0}\right) & =\left(I_{\mathscr{H}}-\frac{x_{0}}{1-x_{0} \bar{z}_{1}^{-1}} u_{1}^{(1)^{*}} K_{1}^{(1)^{-1}} v^{(1)}\right)^{-1}, \\
\widetilde{M}^{(1)}\left(x_{0}\right) & =v^{(1)^{*}}\left(\left|z_{1}\right|^{-2} K_{2}^{(1)}-x_{0}^{-1} K_{1}^{(1)}\right)^{-1} v^{(1)}, \\
\widetilde{L}^{(1)}\left(x_{0}\right) & =\frac{1}{\left|1+x_{0} z_{1}^{-1}\right|^{2}}\left\{u_{2}^{(1)^{*}} K_{2}^{(1)^{-1}} u_{2}^{(1)}-x_{0} u_{1}^{(1)^{*}} K_{1}^{(1)^{-1}} u_{1}^{(1)}\right\}, \\
\widetilde{P}^{(k)}\left(x_{0}\right) & =\left\{I_{\mathscr{H}}-\frac{x_{0}}{1-x_{0} \bar{z}_{k}^{-1}} \widehat{u}_{1}^{(k)^{*}} \widehat{K}_{1}^{(k)^{-1}} \widehat{v}^{(k)}\right\}^{-1}, \\
\widetilde{M}^{(k)}\left(x_{0}\right) & =\widehat{v}^{(k)^{*}}\left(\left|z_{k}\right|^{-2} \widehat{K}_{2}^{(k)}-x_{0}^{-1} \widehat{K}_{1}^{(k)}\right)^{-1} \widehat{v}^{(k)}, \\
\widetilde{L}^{(k)}\left(x_{0}\right) & =\frac{1}{\left|1+x_{0} z_{k}^{-1}\right|^{2}}\left(\widehat{u}_{2}^{(k)^{*}} \widehat{K}_{2}^{(k)^{-1}} \widehat{u}_{2}^{(k)}-x_{0} \widehat{u}_{1}^{(k)^{*}} \widehat{K}_{1}^{(k)^{-1}} \widehat{u}_{1}^{(k)}\right) .
\end{aligned}
$$

Параметры Стилтьеса $L^{(1)}, M^{(1)}$ совпадают с операторами $\widetilde{L}^{(1)}, \widetilde{M}^{(1)}$, а параметры Стилтьеса $L^{(k)}, M^{(k)}$ конгруэнтны операторам $\widetilde{L}^{(k)}, \widetilde{M}^{(k)}$ (см. (31)). Таким образом, мы указали явные формулы для параметров Стилтьеса в задаче Неванлинны-Пика. Теорема 3 задает теперь критерий вполне неопределенности задачи Неванлинны-Пика (41).

4.2. Проблема моментов Стилтьеса. В проблеме моментов Стилтьеса по заданной последовательности операторов $s_{0}, \ldots, s_{k}, \ldots \in\{\mathscr{H}\}_{H}$ требуется описать множество монотонно возрастающих ОФ $\sigma: \mathbb{R}_{+} \rightarrow\{\mathscr{H}\}_{H}, \sigma(0)=0_{\mathscr{H}}, \sigma(t+0)=\sigma(t)$, $t \in[0, \infty)$, таких, что

$$
s_{j}=\int_{0}^{+\infty} t^{j} d \sigma(t) \quad \forall j \in \mathbb{N} \cup\{0\} .
$$

Множество решений $\sigma$ проблемы моментов (48) обозначим символом $\mathscr{M}^{(\infty)}$. С каждой $\sigma \in \mathscr{M}^{(\infty)}$ свяжем ОФ

$$
s(z)=\int_{0}^{+\infty} \frac{d \sigma(t)}{t-z} .
$$

ОФ $s$ определена и голоморфна в $\mathbb{C} \backslash \mathbb{R}_{+}$и называется ассоииированной с проблемой моментов (48). Множество ОФ $s$, ассоциированных с проблемой (48), обозначим символом $\mathscr{F}^{(\infty)}$. Из формулы обращения Стилтьеса следует, что соответствие, устанавливаемое между $\mathscr{F}^{(\infty)}$ и $\mathscr{M}^{(\infty)}$ формулой $(49)$, является взаимно однозначным. Поэтому вместо описания множества $\mathscr{M}^{(\infty)}$ мы можем ограничиться описанием множества $\mathscr{F}(\infty)$.

Вместе с бесконечной проблемой моментов (48) будем рассматривать и усеченные проблемы моментов Стилтьеса. В таких проблемах фиксируется число $l \in \mathbb{N} \cup\{0\}$ и требуется описать все нормированные монотонно возрастающие ОФ $\sigma: \mathbb{R}_{+} \rightarrow\{\mathscr{H}\}_{H}$ и операторы $M \in\{\mathscr{H}\} \geqslant$ такие, что

$$
s_{j}=\int_{0}^{+\infty} t^{j} d \sigma(t), \quad 0 \leqslant j \leqslant l-1, \quad s_{l}=\int_{0}^{+\infty} t^{l} d \sigma(t)+M .
$$

При $l=0$ требуем выполнения только второго условия в (50). 
Проблема моментов (50) называется $l$-й усеченной проблемой моментов $C$ тилmъеса, а множество ее решений $\sigma$ обозначается символом $\mathscr{M}^{(l)}$. Как и в случае проблемы моментов (48), с каждой $\sigma \in \mathscr{M}^{(l)}$ свяжем ассоциированную ОФ $s$ вида (49). Множество всех ОФ $s$, ассоциированных с проблемой $(50)$, обозначим символом $\mathscr{F}^{(l)}$.

Покажем, что задачу описания ассоциированных ОФ можно рассматривать как обобщенную интерполяционную задачу стилтьесовского типа. Рассмотрим отдельно случай четного и нечетного числа моментов.

Пусть $l=2 n, n \in \mathbb{N}$. В этом случае в качестве масштабных пространств выберем пространства

$$
\mathscr{G}_{1}^{(l)}=\underbrace{\mathscr{H} \oplus \mathscr{H} \oplus \cdots \oplus \mathscr{H}}_{n+1}, \quad \mathscr{G}_{2}^{(l)}=\underbrace{\mathscr{H} \oplus \mathscr{H} \oplus \cdots \oplus \mathscr{H}}_{n}, \quad \mathscr{H} .
$$

Операторы $K_{1}^{(l)}, L_{1}^{(l)}, v_{1}^{(l)}, K_{2}^{(l)}, L_{2}^{(l)}, u_{2}^{(l)}$, участвующие в задаче (5), зададим естественными матричными представлениями

$$
\begin{array}{rlrl}
K_{1}^{(l)}=\left\{s_{j+k}\right\}_{j, k=0}^{n}, & L_{1}^{(l)}=\left[\begin{array}{ccc}
I_{\mathscr{H}} & \ldots & 0_{\mathscr{H}} \\
\vdots & \ddots & \vdots \\
0 & \ldots & I_{\mathscr{H}} \\
0 \mathscr{H} & \ldots & 0_{\mathscr{H}}
\end{array}\right], \quad v_{1}^{(l)}=\left[\begin{array}{c}
I_{\mathscr{H}} \\
0_{\mathscr{H}} \\
\vdots \\
0_{\mathscr{H}}
\end{array}\right], \\
K_{2}^{(l)}=\left\{s_{j+k+1}\right\}_{j, k=0}^{n-1}, \quad L_{2}^{(l)}=\left[\begin{array}{ccc}
0 \mathscr{H} & \ldots & 0 \\
I_{\mathscr{H}} & \ldots & 0_{\mathscr{H}} \\
\vdots & \ddots & \vdots \\
0 & \ldots & I_{\mathscr{H}}
\end{array}\right], \quad u_{2}^{(l)}=-\left[\begin{array}{c}
s_{0} \\
s_{1} \\
\vdots \\
s_{n-1}
\end{array}\right] .
\end{array}
$$

Пусть $l=2 n+1, n \in \mathbb{N} \cup\{0\}$. В этом случае в качестве масштабных пространств выберем пространства

$$
\mathscr{G}_{1}^{(l)}=\mathscr{G}_{2}^{(l)}=\underbrace{\mathscr{H} \oplus \mathscr{H} \oplus \cdots \oplus \mathscr{H}}_{n+1}, \quad \mathscr{H} .
$$

Операторы $K_{1}^{(l)}, L_{1}^{(l)}, v_{1}^{(l)}, K_{2}^{(l)}, L_{2}^{(l)}, u_{2}^{(l)}$, участвующие в задаче (5), зададим естественными матричными представлениями

$$
\begin{aligned}
K_{1}^{(l)}=\left\{s_{j+k}\right\}_{j, k=0}^{n}, \quad L_{1}^{(l)}= & {\left[\begin{array}{cccc}
I_{\mathscr{H}} & 0_{\mathscr{H}} & \ldots & 0_{\mathscr{H}} \\
0 & I_{\mathscr{H}} & \ldots & 0_{\mathscr{H}} \\
\vdots & \vdots & \ddots & \vdots \\
0 & 0 & \ldots & I_{\mathscr{H}}
\end{array}\right], \quad v_{1}^{(l)}=\left[\begin{array}{c}
I_{\mathscr{H}} \\
0_{\mathscr{H}} \\
\vdots \\
0_{\mathscr{H}}
\end{array}\right], } \\
K_{2}^{(l)}=\left\{s_{j+k+1}\right\}_{j, k=0}^{n}, \quad L_{2}^{(l)}= & {\left[\begin{array}{cccc}
0_{\mathscr{H}} & \ldots & 0_{\mathscr{H}} & 0_{\mathscr{H}} \\
I_{\mathscr{H}} & \ldots & 0_{\mathscr{H}} & 0_{\mathscr{H}} \\
\vdots & \ddots & \vdots & \vdots \\
0_{\mathscr{H}} & \ldots & I_{\mathscr{H}} & 0_{\mathscr{H}}
\end{array}\right], \quad u_{2}^{(l)}=-\left[\begin{array}{c}
s_{0} \\
s_{1} \\
\vdots \\
s_{n}
\end{array}\right] . }
\end{aligned}
$$

Непосредственно проверяем, что определенные выше операторы при $l=2 n, n \in \mathbb{N}$ и при $l=2 n+1, n \in \mathbb{N} \cup\{0\}$ удовлетворяют ОТ (3). Как известно (см., например, [2]), небходимым и достаточным условием разрешимости задачи (50) являются неравенства $K_{1}^{(l)} \geqslant 0, K_{2}^{(l)} \geqslant 0$. Более того (см. [2]), ОФ $s \in \mathscr{F}^{(l)}$ тогда и только тогда, когда 
она удовлетворяет системе ОМН (6). Таким образом, множество $\mathscr{F}^{(l)}$ совпадает со множеством решений следующей интерполяционной задачи стилтьесовского типа:

$$
\mathscr{P}^{(l)}=\left\{K_{1}^{(l)}, L_{1}^{(l)}, v_{1}^{(l)}, K_{2}^{(l)}, L_{2}^{(l)}, u_{2}^{(l)}\right\} .
$$

Условиями полной неопределенности являются условия $K_{1}^{(l)}>0, K_{2}^{(l)}>0$. Легко видеть, что при этом все остальные условия в (15) автоматически выполнены, т.е. задача (50) является вполне неопределенной.

Пусть задачи (50) являются вполне неопределенными при всех $l$. Интерполяционные задачи (50) являются упорядоченным семейством (см. [7]). Рассмотрим резольвентные матрицы $U_{1}^{(l)}, l \in \mathbb{N} \cup\{0\}$, интерполяционных задач (50) (см. (16)). В [2] показано, что резольвентные матрицы разбиваются в произведение Бляшке-Потапова

$$
\begin{aligned}
U_{1}^{(2 n)}(z) & =\left[\begin{array}{cc}
I & -z M_{0} \\
0 & I
\end{array}\right] \cdot\left[\begin{array}{cc}
I & 0 \\
L_{0} & I
\end{array}\right] \cdots\left[\begin{array}{cc}
I & 0 \\
L_{n-1} & I
\end{array}\right] \cdot\left[\begin{array}{cc}
I & -z M_{n} \\
0 & I
\end{array}\right], \\
U_{1}^{(2 n+1)}(z) & =\left[\begin{array}{cc}
I & -z M_{0} \\
0 & I
\end{array}\right] \cdot\left[\begin{array}{cc}
I & 0 \\
L_{0} & I
\end{array}\right] \cdots\left[\begin{array}{cc}
I & -z M_{n} \\
0 & I
\end{array}\right] \cdot\left[\begin{array}{cc}
I & 0 \\
L_{n} & I
\end{array}\right] .
\end{aligned}
$$

Здесь

$$
\begin{gathered}
M^{(0)}=s_{0}^{-1}>0, \quad L^{(0)}=s_{0} s_{1}^{-1} s_{0}>0 \\
M^{(k)}=v_{1}^{(2 k)^{*}} K_{1}^{(2 k)^{-1}} v_{1}^{(2 k)}-v_{1}^{(2 k-2)^{*}} K_{1}^{(2 k-2)^{-1}} v_{1}^{(2 k-2)}>0, \\
L^{(k)}=u_{2}^{(2 k+1)^{*}} K_{2}^{(2 k+1)^{-1}} u_{2}^{(2 k+1)}-u_{2}^{(2 k-1)^{*}} K_{2}^{(2 k-1)^{-1}} u_{2}^{(2 k-1)}>0,
\end{gathered}
$$

Пусть в формулах (52) z=-1. Из вида формул (52) следует, что операторы в (53) являются обобщенными параметрами Стилтьеса. По теореме 3 проблема моментов (48) вполне неопределена тогда и только тогда, когда сходятся ряды $\sum_{k=0}^{\infty} M^{(k)}$ и $\sum_{k=0}^{\infty} L^{(k)}$.

ЗАмечаниЕ. Из формул (45) и (46) следует, что в задаче Неванлинны-Пика мультипликативное разложение (30) содержит верхние блочно-треугольные, нижние блочно-треугольные и блочно-диагональные операторы. Поэтому обобщенные параметры Стилтьеса $M^{(k)}$ и $L^{(k)}$ конгруэнтны операторам $\widetilde{M}^{(k)}$ и $\widetilde{L}^{(k)}$ (см. формулы (31)). Из формул же (52) следует, что в проблеме моментов мультипликативное разложение (30) содержит только верхние блочно-треугольные и нижние блочно-треугольные операторы. Поэтому обобщенные параметры Стилтьеса $M^{(k)}$ и $L^{(k)}$ coвпадают с операторами $\widetilde{M}^{(k)}$ и $\widetilde{L}^{(k)}$. Таким образом, критерий Стилтьеса в случае проблемы моментов проще, чем аналогичный критерий для задачи Неванлинны-Пика и других интерполяционных задач стилтьесовского типа. Этим, по-видимому, и объясняется тот факт, что в течение более 100 лет со времени работ Стилтьеса [1] критерий Стилтьеса неопределенности проблемы моментов не был обобщен на другие интерполяционные задачи для стилтьесовских функций.

\section{СПИСОК ЦИТИРОВАННОЙ ЛИТЕРАТУРЫ}

[1] T. Stieltjes, "Recherches sur les fractions continues", Ann. Fac. Sci. Toulouse Sci. Math. Sci. Phys., 8:4 (1894), 1-122; 9:1 (1895), 1-47. 
[2] Ю. М. Дюкарев, "О критериях неопределенности матричной проблемы моментов Стилтьеса", Матем. заметки, 75:1 (2004), 71-88.

[3] Н. И. Ахиезер, Классическал проблема моментов и некоторые вопросы анализа, связанные с нею, Физматгиз, М., 1961.

[4] Ю. М. Дюкарев, "Общая схема решения интерполяционных задач в классе Стилтьеса, основанная на согласованных интегральных представлениях пар неотрицательных операторов. I", Матем. физ., анал., геом., 6:1-2 (1999), 30-54.

[5] V. Bolotnikov, L. Sakhnovich, "On an operator approach to interpolation problems for Stieltjes fanctions", Integral Equations Operator Theory, 35:4 (1999), 423-470.

[6] M. G. Krein, A. A. Nudelman, "An interpolation problem in the class of Stieltjes functions and its connections with other problems", Integral Equations Operator Theory, 30:3 (1998), 251-278.

[7] Ю. М. Дюкарев, "О неопределенности интерполяционных задач в классе Стилтьеса", Матем. сб., 196:3 (2005), 61-88.

[8] Ю. М. Дюкарев, "Мультипликативная структура резольвентных матриц интерполяционных задач в классе Стилтьеса", Вісн. Харkіbсъкого ун-ту. Сер. Матем., прикл. матем., мех., 458 (1999), 143-153.

[9] В.П. Потапов, “Дробно-линейные преобразования матриц", Исследования по теории операторов и их приложениям, Наукова думка, Киев, 1979, 75-97.

[10] Ю. М. Дюкарев, "Мультипликативные и аддитивные классы Стилтьеса аналитических матриц-функций и связанные с ними интерполяционные задачи. II", Теория функиий, функи. анализ и их прилож., 1982, № 38, 40-48.

[11] В. П. Потапов, “Теорема о модуле. II”, Теория функиий, функи. анализ и их прилож., 1983, № 39, 95-106.

Ю. М. Дюкарев

Харьковский национальный университет

E-mail: Yury.M.Dyukarev@univer.kharkov.ua
Поступило

10.04.2006

Исправленный вариант

05.02.2007 